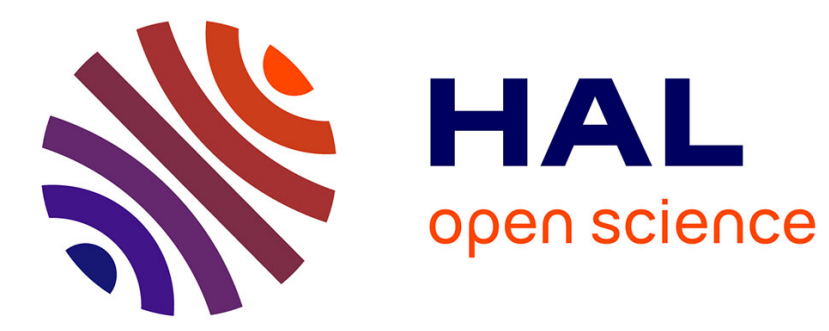

\title{
Progrès récents sur le magnétomètre à vapeur de césium type "asservi"
}

\author{
J.L. Meilleroux
}

\section{To cite this version:}

J.L. Meilleroux. Progrès récents sur le magnétomètre à vapeur de césium type " asservi ". Revue de Physique Appliquée, 1970, 5 (1), pp.121-130. 10.1051/rphysap:0197000501012100 . jpa-00243344

\section{HAL Id: jpa-00243344 https://hal.science/jpa-00243344}

Submitted on 1 Jan 1970

HAL is a multi-disciplinary open access archive for the deposit and dissemination of scientific research documents, whether they are published or not. The documents may come from teaching and research institutions in France or abroad, or from public or private research centers.
L'archive ouverte pluridisciplinaire HAL, est destinée au dépôt et à la diffusion de documents scientifiques de niveau recherche, publiés ou non, émanant des établissements d'enseignement et de recherche français ou étrangers, des laboratoires publics ou privés. 


\title{
PROGRÈS RÉGENTS SUR LE MAGNÉTOMÈTRE A VAPEUR DE GÉSIUM TYPE « ASSERVI »
}

\author{
Par J. L. MEILLEROUX, \\ Thomson/C.S.F., Domaine de Corbeville, Laboratoire Central, 91-Orsay.
}

I. Généralités. - Le principe du magnétomètre à vapeur de césium repose sur la mesure de la fréquence de résonance magnétique de l'atome de césium. Cette fréquence est proportionnelle à la valeur du champ magnétique environnant. L'excitation et la détection de la résonance sont rendues possibles grâce au procédé de pompage optique [1]. L'asservissement d'un générateur au centre de la raie de résonance permet, soit par comptage, soit par discrimination de la fréquence de ce générateur, d'obtenir à chaque instant, soit la valeur absolue, soit les fluctuations du champ magnétique.

Les études sur ce type de magnétomètre ont débuté en 1961 à la C.S.F. (actuellement Thomson-C.S.F.).

Le premier appareil opérationnel a été mis au point en 1963. Il faisait partie d'une première série de cinq qui ont assuré en 1964 le relevé aérien de la carte magnétique de France par la Compagnie Générale de Géophysique avec une précision de l'ordre du gamma $\left(1\right.$ gamma $=10^{-5}$ oersted $)$.

Parallèlement, une version militaire pour la lutte anti-submersible était développée : une série de 70 magnétomètres fut alors construite. Ces appareils équipent actuellement le patrouilleur Bréguet-Atlantic 1150.

Les améliorations apportées depuis 3 ans portent principalement sur :

- les effets d'orientation de la sonde,

- la gamme de fonctionnement en température,

- la technologie de la source de lumière,

- la fiabilité et la miniaturisation des circuits électroniques.

La description complète du fonctionnement du magnétomètre à césium ayant déjà été faite dans un certain nombre de publications [2], [3], nous n'indiquerons dans cet article que les éléments essentiels nécessaires à la compréhension des phénomènes traités.

II. Effets d'orientation de la sonde. - On résume sous l'appellation « effets de rotation » les défauts d'un magnétomètre à champ total qui n'indique pas une valeur constante de champ magnétique lorsqu'on fait varier l'orientation de la sonde détectrice.

Ces effets peuvent être soit dynamiques, soit statiques :

Effets dynamiques : effet gyroscopique, effets dus au courant de Foucault dans les parties métalliques de la sonde.

Effets statiques : magnétisme parasite de la sonde, effets liés au pompage optique.

L'effet gyroscopique est tel que :

$$
\overbrace{\text { Fréquence mesurée }}^{F_{0}=\gamma H_{0}}+\frac{\Omega}{2 \pi}
$$

$\Omega$ : vitesse angulaire de rotation en $\mathrm{rad} / \mathrm{s}$ (le vecteur rotation étant colinéaire à $H_{0}$ ).
La constante $\gamma$ étant égale, dans le cas du césium, à $3,5 \times 10^{5} \mathrm{~Hz}$ par gauss, l'effet gyroscopique sera inférieur à $10^{-6}$ gauss (soit $1 / 10$ de gamma) pour une vitesse de rotation de $2 \mathrm{rad} / \mathrm{s}$. Nous considérerons que, dans la majorité des applications, cette vitesse n'est atteinte que très rarement.

L'effet dû aux courants de Foucault est supprimé en raison de l'absence quasi totale de toute substance métallique au niveau de la sonde.

Le magnétisme parasite de la sonde est négligeable (construction très soignée et mesure du magnétisme résiduel à $0,01 \gamma$ près de tous les composants de la sonde).

Les effets liés au pompage optique sont pratiquement les seuls effets importants. Leur ordre de grandeur est de $2 \gamma$ pour une rotation de $180^{\circ}$ dans le cas le plus défavorable (ce cas défavorable sera explicité par la suite). Ils résultent d'une dissymétrie de la raie de résonance. Pour interpréter cette dissymétrie, nous allons examiner le cycle de pompage optique de l'atome de césium.

II.1. ÉTUDE DE LA DISSYMÉTRIE DE RAIE. - a) Mécanisme de pompage optique et détection optique de la résonance magnétique dans la vapeur de césium. - L'atome de césium possède un diagramme de niveaux d'énergie très

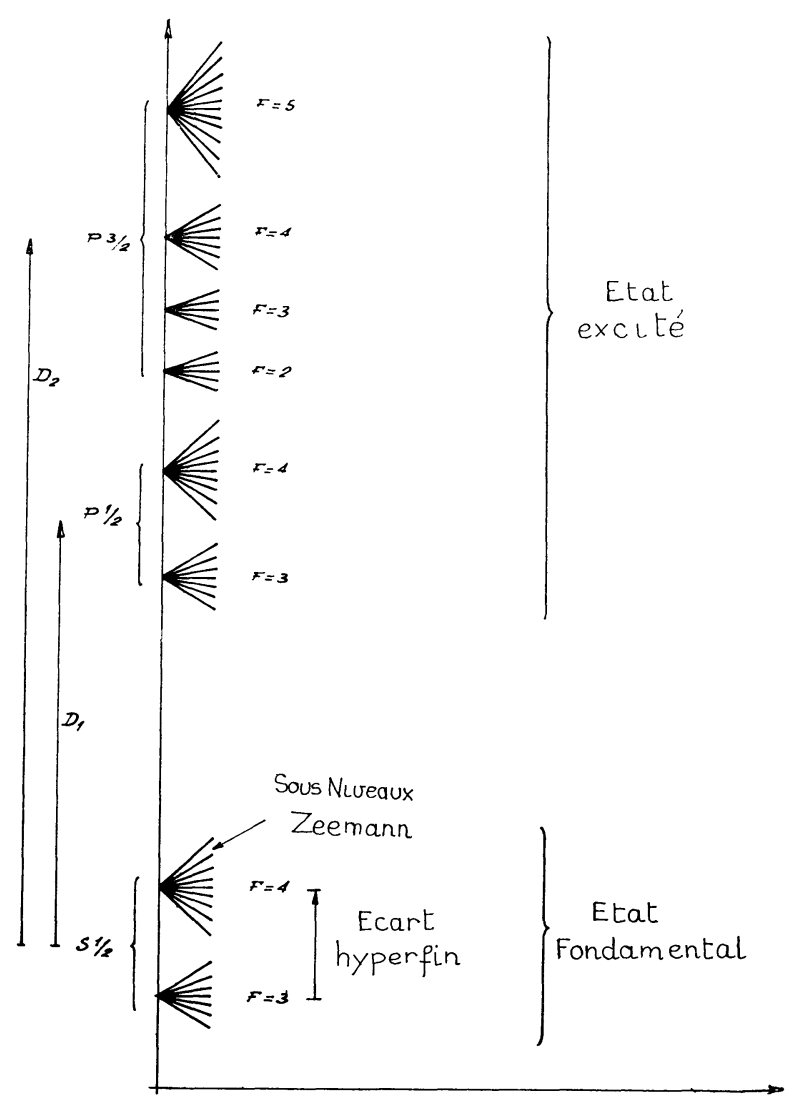

FIG. 1. 
complexe. La figure 1 représente seulement l'état fondamental $S^{1 / 2}$ et les deux états excités $P^{\mathbf{1} / 2}$ et $P^{\mathbf{3} / 2}$.

Pompage optique. - En irradiant la vapeur de césium par la raie $D_{1}$, par exemple, polarisée circulairement, on provoque les transitions du sous-niveau $m$ de l'état fondamental au sous-niveau $m+1$ de l'état excité. L'atome séjourne un certain temps dans l'état excité avant de retomber sur l'état fondamental $m, m+1$ ou $m+2$.

Si le processus dure suffisamment longtemps, les atomes qui occupaient initialement tous les sousniveaux avec la même répartition, seront pratiquement tous groupés dans le sous-niveau $m$ le plus élevé (niveau fondamental).

On dit alors que la vapeur est « pompée ». L'absorption de la radiation polarisée circulairement par la vapeur dépendant des populations de chaque sousniveau, la vapeur est plus transparente lorsque les atomes sont dans l'état « pompé » que lorsqu'ils sont à l'équilibre thermique.

$\mathrm{Si}$ on applique maintenant une onde électromagnétique dont la fréquence correspond à l'écart énergétique de deux sous-niveaux, les atomes transiteront d'un sous-niveau $m$ au sous-niveau $m-1$ : on a résonance.

Détection optique de la résonance. - L'établissement de la résonance s'accompagne donc d'une égalisation des populations des sous-niveaux; en conséquence, la vapeur de césium devient moins transparente à la radiation lumineuse et cette diminution de lumière, observée par une cellule photoélectrique par exemple, permet de détecter l'état de résonance. La courbe de la figure 2 traduit la variation du courant de la cellule d'observation en fonction de la fréquence de l'onde électromagnétique.

Ce procédé de détection est dû au physicien Dehmelt. On utilise également la détection de lumière " diffusée »: en effet, les atomes de césium irradiés par la source de lumière polarisée émettent également une lumière de résonance qui tend vers zéro lorsque la vapeur est pompée. Lorsqu'on excite la résonance radiofréquence, la lumière de résonance augmente et passe par un maximum au centre de la raie [1].

Cette lumière est captée par une mosaïque de cellules photos entourant la cellule de résonance [4]. Ce procédé de détection donne les résultats légèrement supérieurs en ce qui concerne le rapport signal sur bruit (les cellules photos fonctionnent à courant plus faible).

Cependant, pour une question de simplicité de schémas, nous raisonnerons sur la détection par absorption, les résultats expérimentaux étant identiques.

b) Relation entre $H_{0}$ champ magnétique et $f_{0}$ fréquence de résonance. - Dans un champ $H_{0}$, l'énergie $E_{m}$ d'un sous-niveau Zeeman est donnée par la formule de Breit Rabi.

Cas du niveau $F=4\left({ }^{1}\right)$ :

$$
\begin{aligned}
E_{m}=\frac{-\Delta E_{0}}{2(2 i+1)} & +g_{i} \mu_{B} m H_{0} \\
& +\frac{\Delta E_{0}}{2}\left(1+\frac{4 m}{2 i+1} x+x^{2}\right)^{1 / 2}
\end{aligned}
$$

(1) Seul le niveau $F=4$ sera considéré par la suite, le niveau $F=3$ n'étant pas utilisé dans le magnétomètre (signal de résonance plus faible).

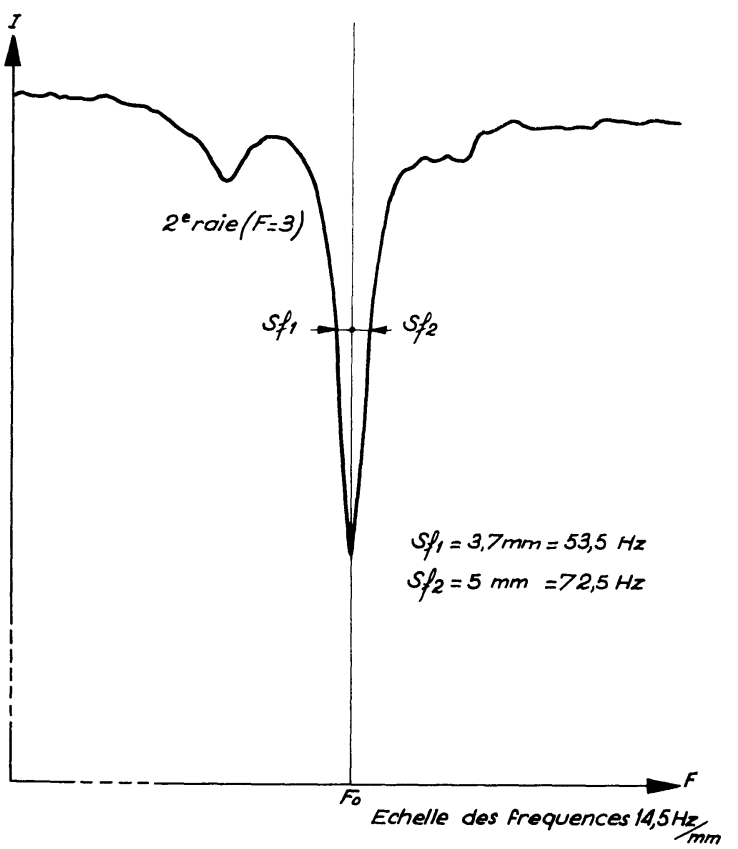

Courbe a. - Enregistrement d'une raie de résonance avec forte lumière et faible champ $H_{1}(10 \gamma)$ :

$$
\begin{aligned}
& \delta f_{1}=3,7 \mathrm{~mm}=53,5 \mathrm{~Hz} \\
& \delta f_{2}=5 \quad \mathrm{~mm}=72,5 \mathrm{~Hz}
\end{aligned}
$$

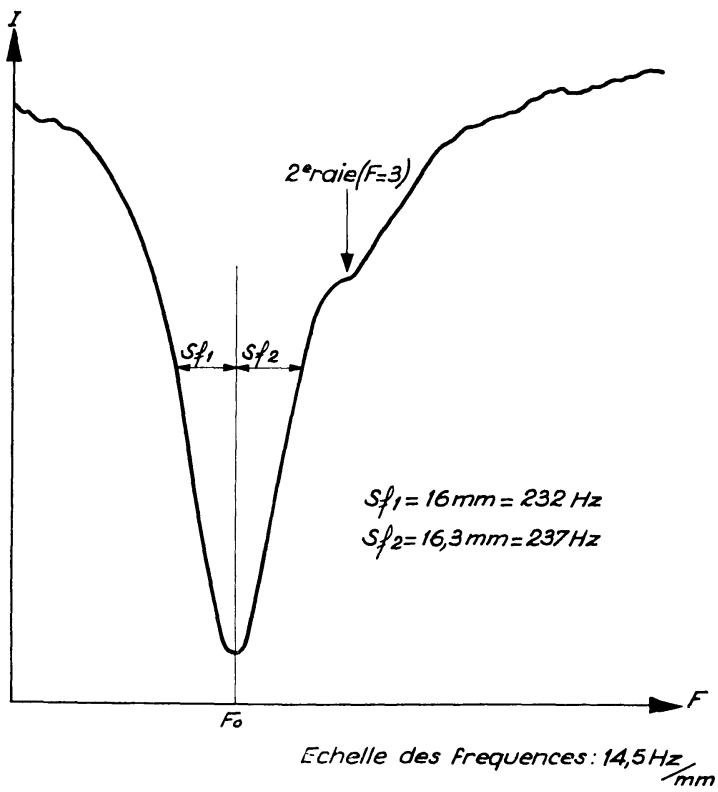

Courbe b. - Enregistrement d'une raie de résonance avec forte lumière et fort champ $H_{1}(50 \gamma)$ :

$$
\begin{aligned}
& \delta f_{1}=16 \mathrm{~mm}=232 \mathrm{~Hz} \\
& \delta f_{2}=16,3 \mathrm{~mm}=237 \mathrm{~Hz} .
\end{aligned}
$$

FIG. 2.

avec $m$ pouvant prendre les valeurs : $-4,-3,-2$, $-1,2,3,4$; soit 9 sous-niveaux; $\Delta E_{0}$, écart hyperfin en champ nul; $i$, spin nucléaire (ici $7 / 2$ ); $\mu_{B}$, magnéton de Bohr; $g_{j}$, facteur de Landé électronique; $g_{i}$, facteur de Landé nucléaire.

$$
x=\frac{g_{j}-g_{i}}{\Delta E_{0}} \mu_{B} H_{0} .
$$

En considérant $x$ petit (champ faible), on peut écrire l'écart énergétique entre deux sous-niveaux de la façon suivante : 


$$
\begin{aligned}
E_{m}-E_{m-1}=\frac{g_{j} \mu_{B} H_{0}}{2 i+1}+ & \frac{2 i}{2 i+1} g_{i} \mu_{B} H_{0} \\
& -\frac{(2 m-1)}{(2 i+1)^{2}} \frac{g_{j}^{2} \mu_{B}^{2} H_{0}^{2}}{\Delta E_{0}} .
\end{aligned}
$$

On voit sur cette formule que l'écart $E_{m}-E_{m-1}$ dépend de $m$ dans le coefficient de $H_{0}^{2}$.

Les écarts énergétiques entre les niveaux $m$ adjacents ne sont donc pas égaux, ce qui signifie (chaque écart donnant lieu à une raie de résonance) que les huit raies de résonance correspondant aux neuf sousniveaux sont décalées dans l'échelle des fréquences.

L'écart entre chaque raie peut être calculé au moyen de la formule (2). On trouve dans un champ

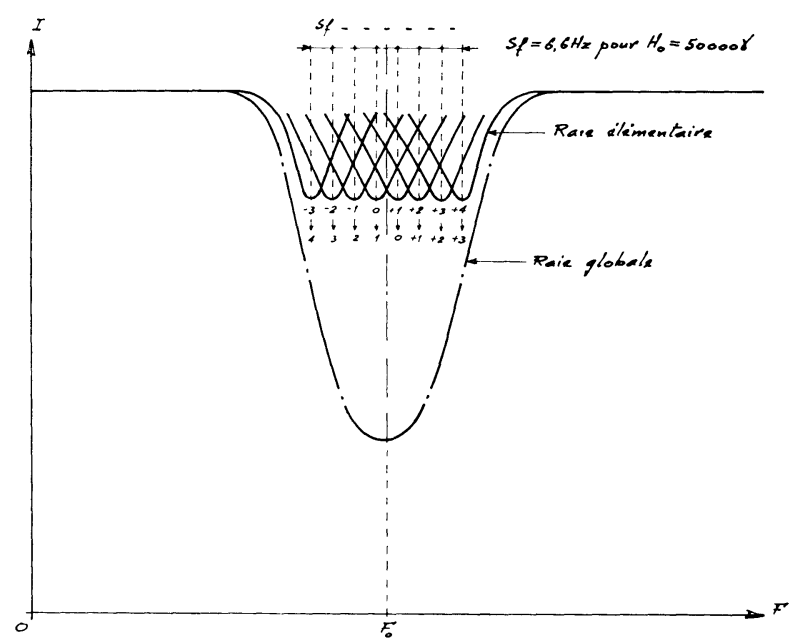

FIG. 3.

de $50000 \gamma$ un écart de $6,6 \mathrm{~Hz}$ en exprimant $E_{m}-E_{m-1}$ en unités de fréquence.

Cet écart étant inférieur à chaque largeur de raie déterminée par les constantes de temps de relaxation du système d'atomes, le spectre n'est pas résolu et l'on observe une raie de résonance globale, somme de ces huit raies élémentaires.

Si ces huit raies élémentaires étaient égales, on obtiendrait une raie composite dont la fréquence $F_{0}$ correspondrait à la moyenne des fréquences de ces raies (fig. 3).

$$
F_{0}=\gamma \times H_{0}
$$

$\gamma$ : constante gyromagnétique du césium $(\gamma=3,5 \mathrm{~Hz}$ par gamma, valeur moyenne calculée à partir de la formule (2)).

c) Dissymétrie de la raie de résonance. - D'après le principe même du pompage optique qui tend à accumuler les atomes dans l'état $m$ le plus élevé (lorsque la lumière est de polarisation circulaire droite), on conçoit aisément que les huit raies élémentaires ne peuvent être égales. Supposons en effet que tous les atomes soient dans l'état $m=+4$. Les atomes, sous l'effet du champ radioélectrique de résonance, transiteront de +4 à +3 , puis de +3 à +2 , etc. Mais parallèlement à ces transitions, le pompage optique aura tendance à remonter les atomes vers $m=+4$. Les raies de résonance concernant les $m \rightarrow m-1$ décroissants auront donc de moins en moins d'amplitude.

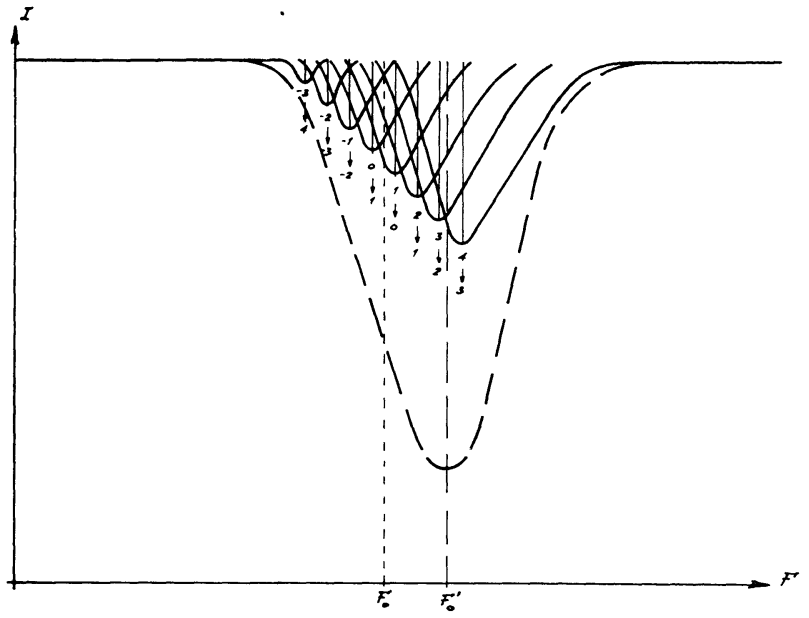

FIG. 4.

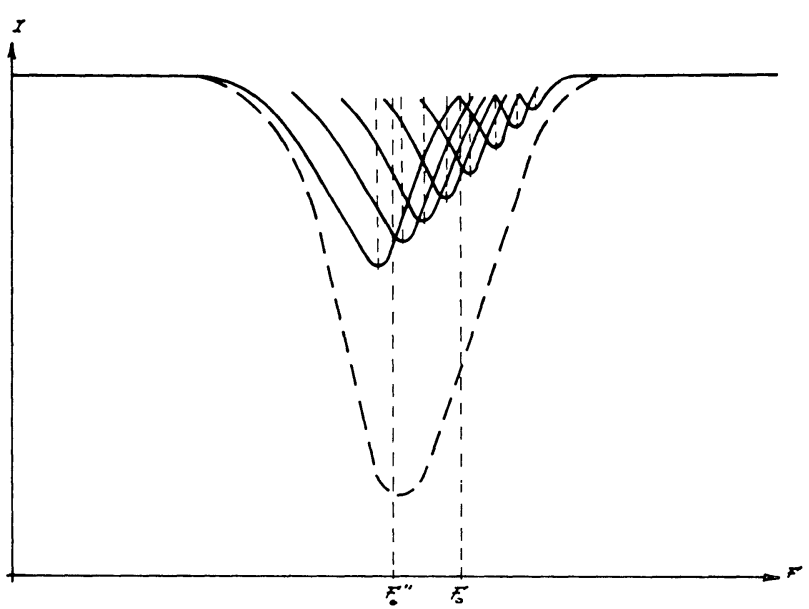

FIG. 5.

Finalement, la raie globale de résonance prendrá l'allure indiquée sur la figure 4 .

La fréquence centrale de la raie globale deviendra différente de $F_{0}$.

Si l'on inverse le sens de la polarisation circulaire de la lumière, on tendra à accumuler les atomes dans l'état $m$ le moins élevé $(m=-4)$. En reprenant le même raisonnement que ci-dessus, on constate que la raie globale de résonance prend la dissymétrie inverse de la précédente ( fig. 5).

Le sens de la polarisation circulaire de la lumière est défini par rapport à la direction du champ $H_{0}$.

En particulier, inverser le sens de $H_{0}$ revient à inverser le sens de la polarisation de la lumière ainsi défini.

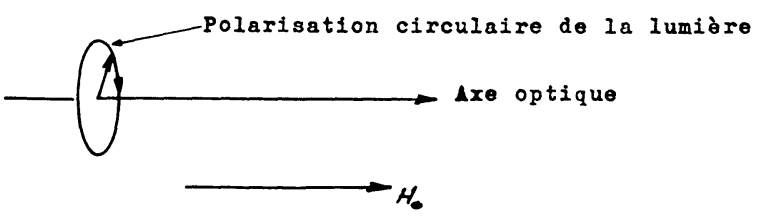

FIG. 6 .

Ainsi, lorsqu'on retourne une sonde de magnétomètre dans le champ, le sens de polarisation de lumière s'inverse par rapport au champ et le sommet de la raie 
de résonance varie de $F_{0}^{\prime}$ à $F_{0}^{\prime \prime}$ (cf. fig. 4 et fig. 5).

Il en résulte un effet de rotation dont on peut calculer la limite supérieure à l'aide de la formule (1).

On trouve, pour les champs faibles :

$$
2 \times \frac{F_{\max }-F_{\min }}{F_{\max }+F_{\min }}=2 \frac{g_{j}-g_{i}}{\Delta E_{0}} \mu_{B} H_{0} .
$$

Pour le Cs $\left\{\begin{aligned} & F_{\max } \text { correspond à la transition } \\ & m=+4 \rightarrow m=+3 \\ & F_{\min } \text { correspond à la transition } m=-3 \rightarrow m=-4 .\end{aligned}\right.$

L'aspect de cette formule est assez remarquable : la valeur théorique de l'effet de rotation ne dépend pas du spin nucléaire, mais ne dépend que de l'écart hyperfin.

En exprimant $H$ en gauss, le coefficient numérique vaut :

$$
\frac{\Delta F}{F}=2 \frac{F_{\max }-F_{\min }}{F_{\max }+F_{\min }}=6 \times 10^{-4} H_{0} \text { gauss. }
$$

En effectuant le calcul pour les autres alcalins, on trouve :

$$
\begin{array}{lrl}
R_{b} 85 & 18,6 & \times 10^{-4} H \text { gauss } \\
R_{b} 87 & 8,2 & \times 10^{-4} H \text { gauss } \\
K & 220 & \times 10^{-4} H \text { gauss. }
\end{array}
$$

Cet effet de rotation théorique correspond au cas limite où la lumière de pompage serait infinie et où l'intensité de champ électromagnétique tendrait vers zéro. En effet, ne sont considérées alors que les transitions $+4 \rightarrow+3$ dans un cas et $-3 \rightarrow-4$ dans l'autre.

Dans la pratique, toutes les transitions existent et les raies de résonance ont effectivement le profil représenté sur les figures 4 et 5 , mais avec une dissymétrie beaucoup plus faible et dépendant, comme l'indique qualitativement la théorie, de la lumière et du champ électromagnétique.

II.2. ÉTUDE EXPÉRIMENTALE DE LA DISSYMÉTRIE DE RAIE. - A. Description de l'expérience. - Un générateur calé à $F_{0}(\mathrm{kHz})$ excite l'enroulement $\mathrm{HF}$ d'une sonde de magnétomètre. Un générateur injecte un courant

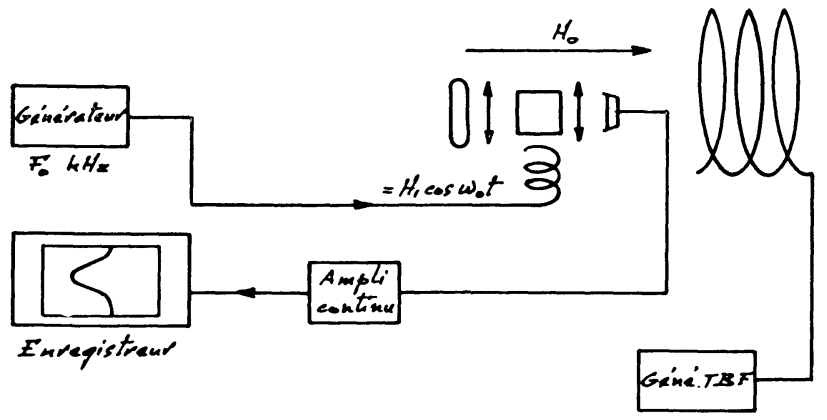

FIG. 7.

lentement variable (linéairement avec le temps) dans des bobines Helmholtz entourant la sonde, de façon à faire varier le champ total $H$ de $H_{0}-\Delta H$ à $H_{0}+\Delta H$.
En enregistrant la tension de sortie de la cellule photo (après l'avoir amplifiée dans une bande de 0 à $1 \mathrm{~Hz}$ ), on obtient directement la courbe d'absorption réelle due au passage à la résonance pour :

$$
F_{0}=3,5 \times 10^{5} H_{0} \text { en } \mathrm{Hz} / \text { gauss. }
$$

B. Mesure de la dissymétrie de raie. - On a effectué un grand nombre d'enregistrements de raies pour les valeurs de $H_{1}$ variant de 10 à $50 \gamma$.

Des études précédentes [5] ont montré que c'est à l'intérieur de cette plage que l'on obtient les signaux à bruit optimum et des largeurs de raie suffisamment étroites. Deux exemples d'enregistrement de raies de résonance sont donnés ci-contre.

Les dissymétries de raie sont mesurées de la façon suivante :

— on exprime les demi-largeurs à $6 \mathrm{~dB}$, soit $\delta f_{1}$ et $\delta f_{2}$.

Le rapport $\frac{\delta f_{1}-\delta f_{2}}{\delta f_{1}+\delta f_{2}}$ représente l'écart du sommet au centre de la raie, écart rapporté à la demi-largeur de raie moyenne.

On a tracé ci-contre : $D=\frac{\delta f_{1}-\delta f_{2}}{\delta f_{1}+\delta f_{2}}=f\left(H_{1}\right)$. Cette courbe confirme bien la théorie : les raies se symétrisent lorsque l'on tend à saturer la résonance en augmentant $H_{1}$ à lumière constante.

II.3. EFFETS DE ROTATION MESURÉs (sonde à faisceau unique). - On fait tourner la sonde autour de l'axe du ballon de résonance, l'axe optique restant dans le plan vertical contenant $H_{0}$, c'est-à-dire le plan nordsud.

Un magnétomètre de référence est utilisé en permanence pendant la durée de la rotation et on porte la différence $\Delta H$ des indications de ces deux magnétomètres en fonction de l'angle de rotation de la sonde du premier.

La mesure se fait au moyen de deux compteurs de fréquence synchronisés. Chacune des fréquences issues des magnétomètres a été auparavant multipliée par 28, ce qui donne approximativement, la dernière décimale lue correspondant au 1/100 de gamma :

$28 \times F_{0} \mathrm{~Hz}=28 \times 3,5 H$ gammas $\simeq 100 \times H$ gammas.

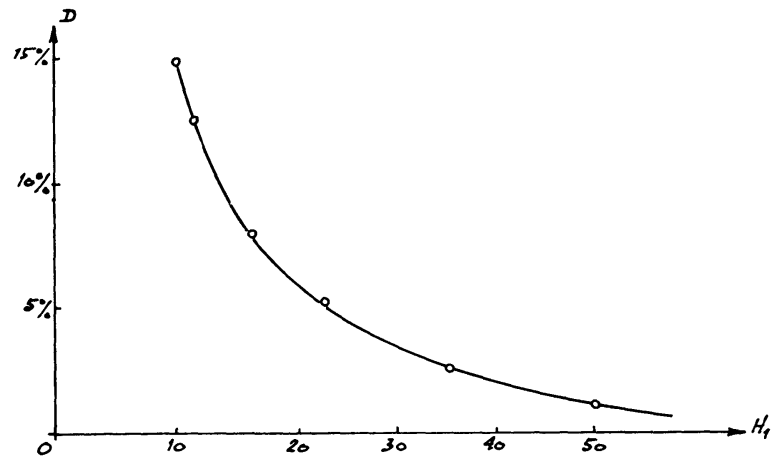

FIG. 8.

Les courbes de la figure 10 donnent les variations de $\Delta H$ en lumière circulaire droite et lumière circulaire gauche pour deux valeurs de $H_{1}$. 

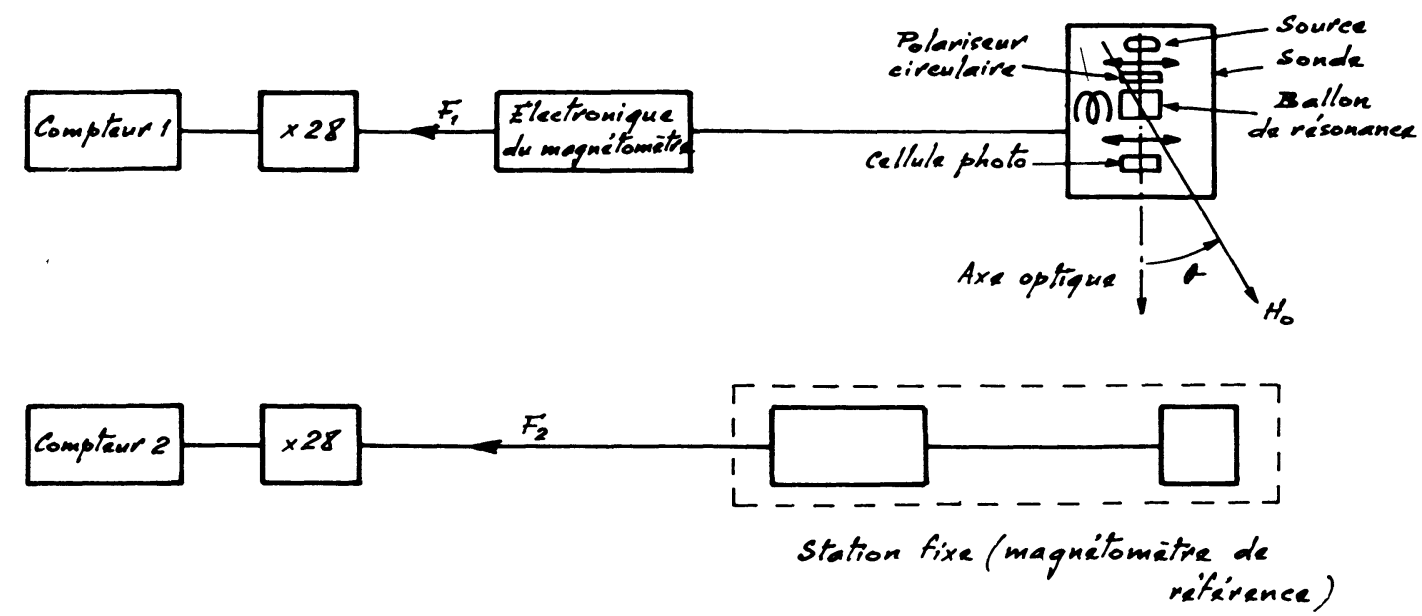

FIG. 9 .

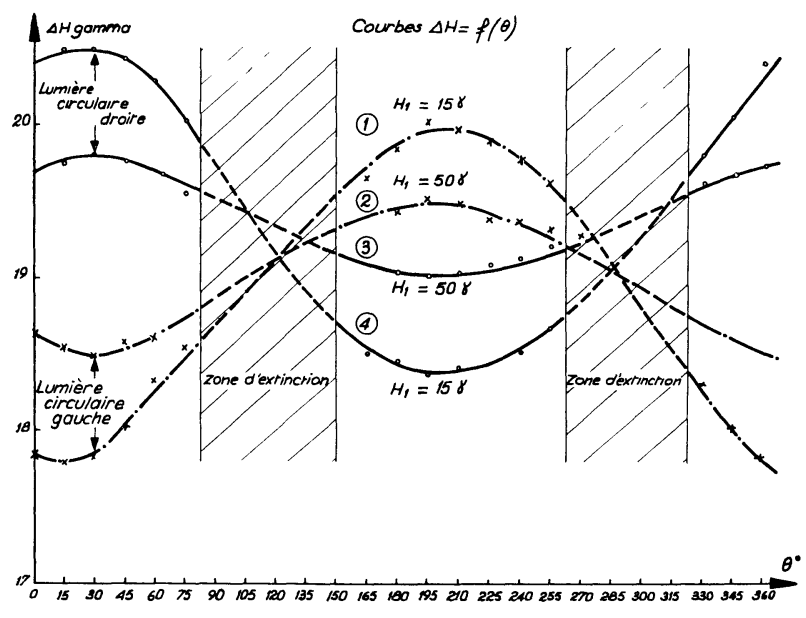

FIG. 10. - L'axe optique est aligné avec le champ $H_{0}$ pour $\theta=25^{\circ}$ et $\theta=205^{\circ}$.

Les courbes (1), (2), (3) et (4) confirment remarquablement les hypothèses théoriques :

- Les diagrammes (1) et (4), tracés avec des lumières de polarisation opposée, sont effectivement complémentaires : l'addition de ces diagrammes (qui pourra être obtenue en mettant en série deux voies de pompage ainsi opposées sous réserve de l'identité rigoureuse de ces voies) donnera un diagramme résultant pratiquement « plat ».

- On constate l'influence de l'augmentation de $H_{1}$ sur l'amplitude crête à crête : celle-ci diminue lorsque $H_{1}$ augmente, ce qui rend bien compte de la symétrisation de la raie de résonance.

- On sait cependant que le rapport signal à bruit se détériore très vite lorsque $H_{1}$ croît, l'optimum se situant pour $H_{1} \simeq 15 \gamma$. Aussi ne peut-on pas diminuer les effets de rotation en agissant sur ce paramètre.

II.4. RÉAlisation D'UNE SONDE A EFFET DE ROTATION RÉDUIT. - La solution choisie a donc été la suivante : addition des signaux de résonance provenant de deux voies de pompage optique de polarisation opposée ( $f g$. 11).

Une « balance » des deux voies a été introduite pour tenir compte de la dispersion éventuelle des éléments de chaque voie (cellule de résonance, polariseur, cellules photos).

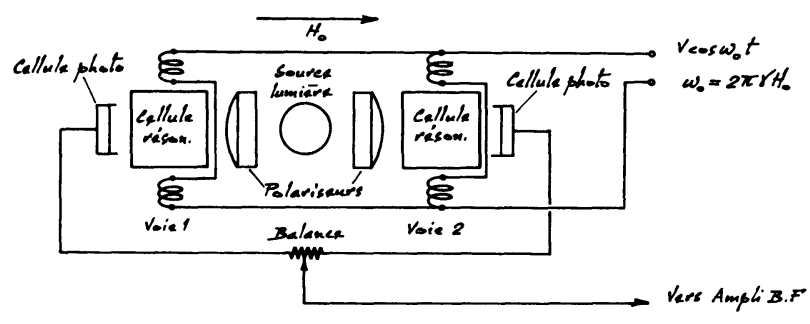

FIG. 11.

En effet, si l'on appelle $\Delta f_{1}$ et $\Delta f_{2}$ les valeurs de l'écart de fréquence par rapport à la fréquence moyenne pour les voies 1 et 2 et $a_{1}$ à $a_{2}$, les signaux de résonance respectifs, $\Delta f$ écart résultant peut s'écrire :

$$
\Delta f=\frac{a_{1} \Delta f_{1}+a_{2} \Delta f_{2}}{a_{1}+a_{2}}
$$

$a_{1}$ : signal de la voie 1 ,

$a_{2}$ : signal de la voie 2 .

En admettant pour $\Delta f_{1}$ et $\Delta f_{2}$ des lois sinusoïdales en fonction de $\theta$ l'angle de rotation (hypothèse inspirée des diagrammes expérimentaux), on obtient :

$$
\begin{aligned}
& \Delta f=\frac{a_{1} \Delta F_{1} \cos \theta+a_{2} \Delta F_{2} \cos (\theta+\pi)}{a_{1}+a_{2}} \\
& \Delta f=\frac{\left(a_{1} \Delta F_{1}-a_{2} \Delta F_{2}\right) \cos \theta}{a_{1}+a_{2}}
\end{aligned}
$$

avec $\Delta F_{1}$ et $\Delta F_{2}$ les amplitudes des diagrammes de rotation des voies 1 et 2 :

$$
\Delta F=\frac{\Delta F_{1}-\Delta F_{2}}{2}+\frac{\Delta F_{1}+\Delta F_{2}}{2} \frac{a_{1}-a_{2}}{a_{1}+a_{2}} .
$$

Supposons :

$$
\begin{aligned}
& \Delta F_{1}=\Delta F_{2}=\Delta F_{0} \\
& \Delta F=\Delta F_{0} \frac{a_{1}-a_{2}}{a_{1}+a_{2}} .
\end{aligned}
$$

Si on veut obtenir une réduction d'un facteur 10 sur $\Delta F_{0}$, on voit qu'il faut que :

$$
\frac{a_{1}-a_{2}}{a_{1}+a_{2}}<\frac{1}{10}
$$

soit $\rho$ le rapport des signaux $\rho=\frac{a_{2}}{a_{1}}, \frac{1-\rho}{1+\rho}<\frac{1}{10}$,
$1>\rho>0,8$. 
La réalisation définitive de la sonde ne pouvait pas cependant être aussi simple, car une structure telle que celle de la figure 11 présente une zone d'extinction de signal : en pratique, si le champ $H_{0}$ se trouve en dehors d'un cône d'axe colinéaire à l'axe optique et de demi-angle d'ouverture égal à $50^{\circ}$, le signal à bruit devient insuffisant.

On est donc conduit :

- soit à disposer un deuxième ensemble de deux voies opposées d'axe perpendiculaire au premier (sonde à 4 faisceaux). Ceci permet d'obtenir un fonctionnement correct quelle que soit l'inclinaison du champ à condition de placer cette sonde dans deux positions distinctes suivant la latitude : plan des axes optiques horizontal de l'équateur à la latitude $45^{\circ}$, plan des axes optiques vertical de la latitude $45^{\circ}$ au pôle,

- soit à disposer deux autres ensembles de deux voies opposées tels que les 3 axes optiques forment un trièdre tri-rectangle (sonde à 6 faisceaux). Cette sonde est alors omnidirectionnelle.

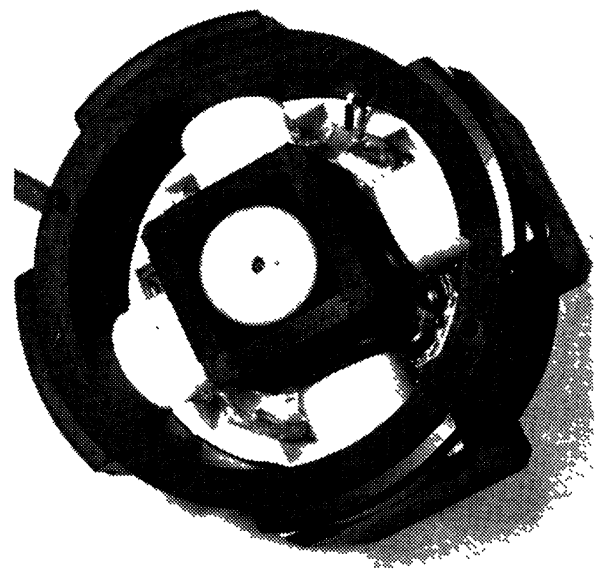

Рното 1. - Sonde à 4 faisceaux.

Dans bien des cas, la première solution est suffisante : c'est la raison pour laquelle nous avons construit une série de mágnétomètres à 4 faisceaux (photo 1 ).

Les résultats obtenus en rotation sont illustrés par la figure 12 .

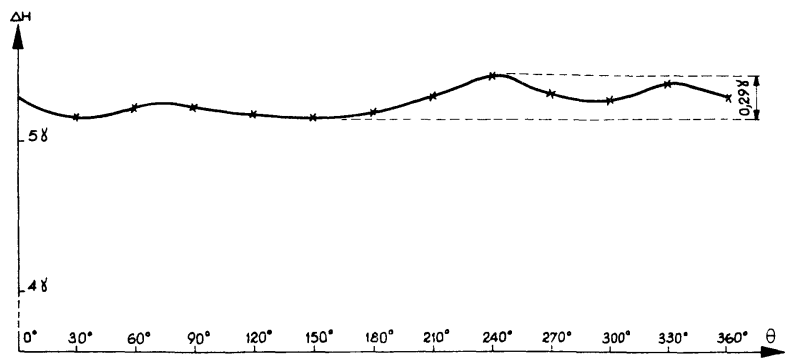

FIG. 12. - Diagramme de rotation.

L'amplitude crête à crête est inférieure à $0,3 \gamma$ et la pente maximum est inférieure à $0,003 \gamma$ par degré.

Cependant, nous réalisons actuellement, pour supprimer la sujétion des deux positions de sonde suivant les zones de latitude, une sonde à 6 faisceaux dont la structure est indiquée sur la photo 2 . On voit sur cette photo la source de lumière à demi-sortie.

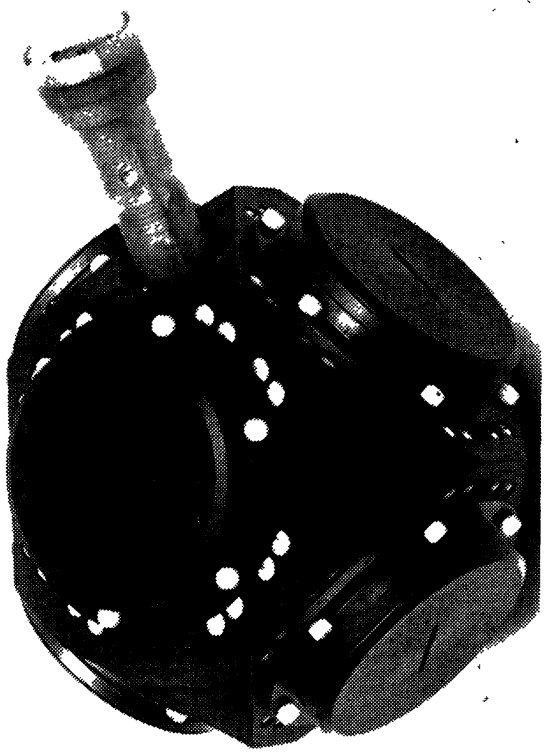

Phoтo 2. - Sonde à 6 faisceaux.

Cette source de lumière, pratiquement identique à celle des sondes à 4 faisceaux, est décrite au $\S$ IV.

III. Élargissement de la gamme de température. La gamme de température de fonctionnement des magnétomètres à vapeur de césium s'étendait initialement de $-20^{\circ} \mathrm{C}$ à $+40^{\circ} \mathrm{C}$.

La limite basse était conditionnée par certains circuits (principalement l'oscillateur d'excitation de la source de lumière), la limite haute était conditionnée par la densité d'atomes de Cs maximum que l'on pouvait admettre dans la cellule de résonance.

La limite basse a été portée de $-20^{\circ} \mathrm{C}$ à $-40^{\circ} \mathrm{C}$ grâce aux progrès réalisés en particulier sur les transistors V.H.F. de puissance (cf. $\S$ IV).

L'extension de la limite haute a été obtenue en choisissant à la fois les dimensions de la cellule de résonance et un gaz tampon approprié sous une pression déterminée.

Nous allons tout d'abord mettre en évidence qualitativement la raison pour laquelle il existe une densité d'atome de Cs optimum, puis expliquer les solutions permettant d'augmenter cette densité, c'est-à-dire augmenter la température maximum de fonctionnement puisque le césium contenu dans la cellule est sous forme de vapeur.

III.1. EXISTENGE D'UN OPTIMUM DE TEMPÉRATURE. - On a vu au § II.1 a) que la vapeur de césium devient plus transparente lorsqu'elle est irradiée par la raie $D_{1}$ ou $D_{2}$ polarisée circulairement. Cependant il existe alors une certaine absorption $\Delta I_{0}$ ( fig. 13).

Cette courbe représente la raie d'émission de la source de lumière creusée en son centre par l'absorption de la vapeur (la largeur de la raie émise est beaucoup plus importante que la largeur de la raie d'absorption).

L'application du champ radiofréquence de résonance a pour effet d'augmenter cette absorption et le signal de résonance est proportionnel à cette augmentation d'absorption.

Lorsque la température de la cellule est faible, il y a peu d'atomes, donc peu d'absorption et le signal de résonance est faible. 


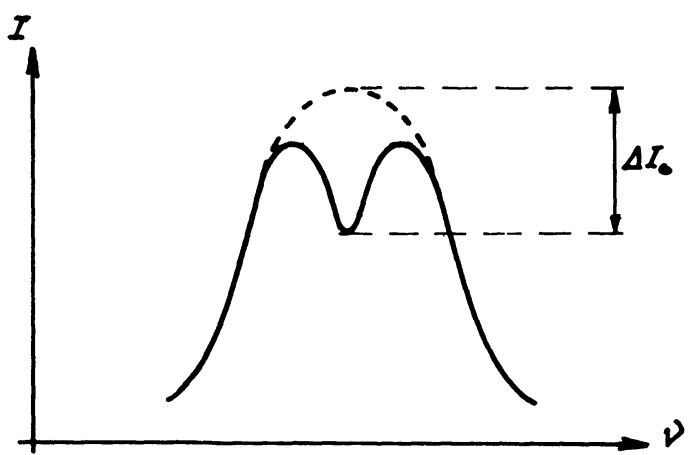

FIG. 13.

Lorsque la température croît, l'absorption augmente et le signal augmente également. Mais l'absorption devient forte, il y a saturation et il devient impossible de déceler la variation d'absorption due à la résonance.

Les figures 14 a, 14 b et 14 c illustrent la progression du phénomène.

On remarque que l'absorption supplémentaire due à la résonance, représentée par la surface noircie, commence par croître avec la température en même temps que l'absorption permanente, pour passer par un maximum, mais pour une température trop élevée, la surface noircie diminue au point de disparaître.

Si on parvient à élargir la raie d'absorption optique du césium, la lumière de pompage sera moins profondément creusée pour une absorption donnée, donc aussi pour une température donnée. Ceci est illustré par les deux figures 15 a et $15 \mathrm{~b}$.

On remarque que pour une absorption optique donnée, représentée par une surface hachurée déterminée, le fait d'élargir la raie d'absorption en diminue la profondeur.

Par conséquent, l'absorption supplémentaire à la résonance (surface noircie) reprendra une amplitude proportionnelle à l'absorption permanente et on pourra atteindre un nouvel optimum, selon un cas de figure analogue à la figure $14 \mathrm{~b}$, mais à température plus élevée.

III .2. Solution Adoptée. - Afin d'élargir la raie d'absorption optique du césium, il faut réduire la durée de vie des atomes dans l'état excité. Une solution consiste à introduire un gaz étranger dans le ballon, le résultat cherché étant obtenu grâce aux collisions des atomes d'alcalin avec le gaz.

Ces collisions présentent un inconvénient : les atomes se répartissant uniformément entre les différents
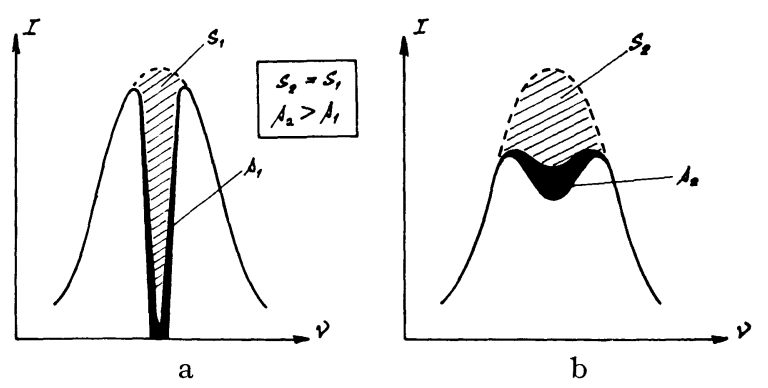

FIG. 15.

a) Absorption de la lumière de pompage : cas habituel, à une température $T_{1}>T$ optimale.

b) Avec raie d'absorption élargie, à la même température $T_{1}$.

niveaux d'énergie de l'état excité avant de retomber à l'état fondamental, le pompage de type Kastler utilisé sur les magnétomètres devient inefficace, les atomes de césium retombant sur n'importe quel niveau Zeeman de l'état fondamental avec suppression de « l'orientation » de la vapeur alcaline. On peut cependant réaliser une différence de population dans l'état fondamental en pompant les niveaux de cet état avec des vitesses différentes, en utilisant, soit la raie $D_{1}$ seule, soit $\mathrm{D}_{2}$ : c'est le pompage de type Dehmelt. Il est donc nécessaire de supprimer l'une de ces deux raies en utilisant un filtre interférentiel $\left({ }^{2}\right)$.

Après expérimentation sur différents gaz, notre choix s'est porté sur l'azote. Pour une cellule de $20 \mathrm{~mm}$ d'épaisseur, la courbe de la figure 16 montre la variation de la température optimum en fonction de la pression.

La pression adoptée finalement est de $3 \mathrm{~cm}$ de mercure.

L'azote convient particulièrement bien pour la détection sur la lumière absorbée.

Cependant, les collisions des atomes de césium dans l'état excité avec les molécules diatomiques d'azote sont du type « de deuxième espèce » : au cours d'un choc, l'énergie d'un atome alcalin est cédée à une molécule d'azote sous forme d'énergie cinétique, et par suite le retour de l'atome dans l'état fondamental s'effectue sans rayonnement : il n'y a pas de lumière de fluorescence. Ce phénomène porte le nom de «Quenching ».

(2) Nous avons actuellement mis au point un autre type de pompage qui permet, dans ce cas également, de s'affranchir du filtre interférentiel : le brevet est en cours de dépôt.

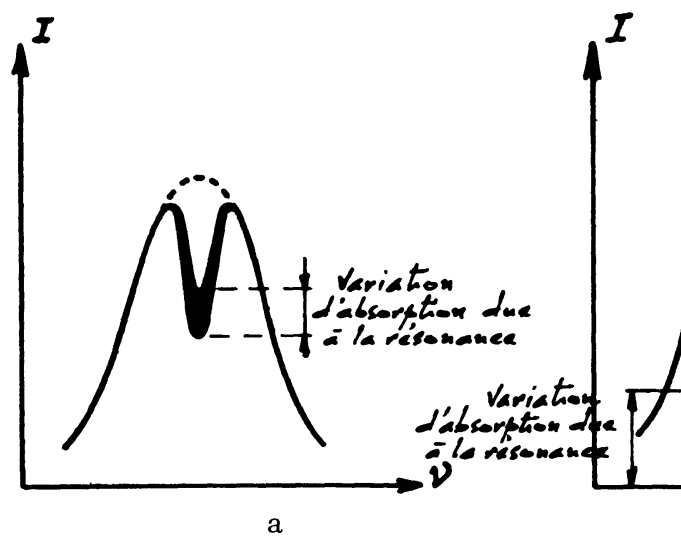

FIG. 14. - a) Température basse ; b) $T^{\text {o }}$ optimum ;

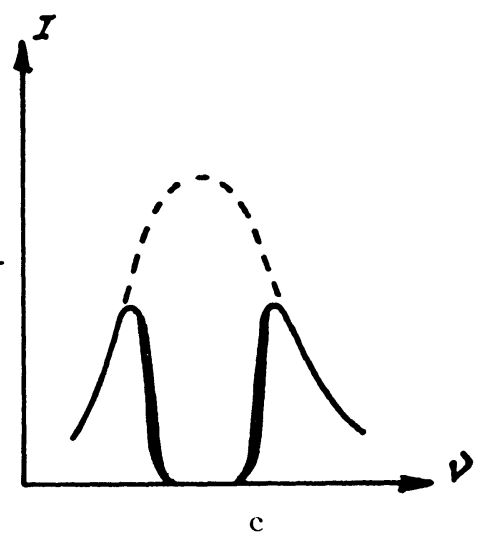

c) To trop élevée. 
En conséquence, l'azote n'est pas utilisable dans le cas de la détection en lumière diffusée ( $\S$ II.1 a).

On utilise alors le néon sous forte pression qui donne des résultats assez voisins.

III.3. Extension JusQu'a $80^{\circ} \mathrm{C}$ de la gamme DE TEMPÉRATURE. - Une étude sur les alliages alcalins [5] a permis d'obtenir une tension de vapeur sensiblement abaissée : à titre d'exemple un alliage rubidium-potassium permet d'obtenir à $70^{\circ} \mathrm{C}$ la même

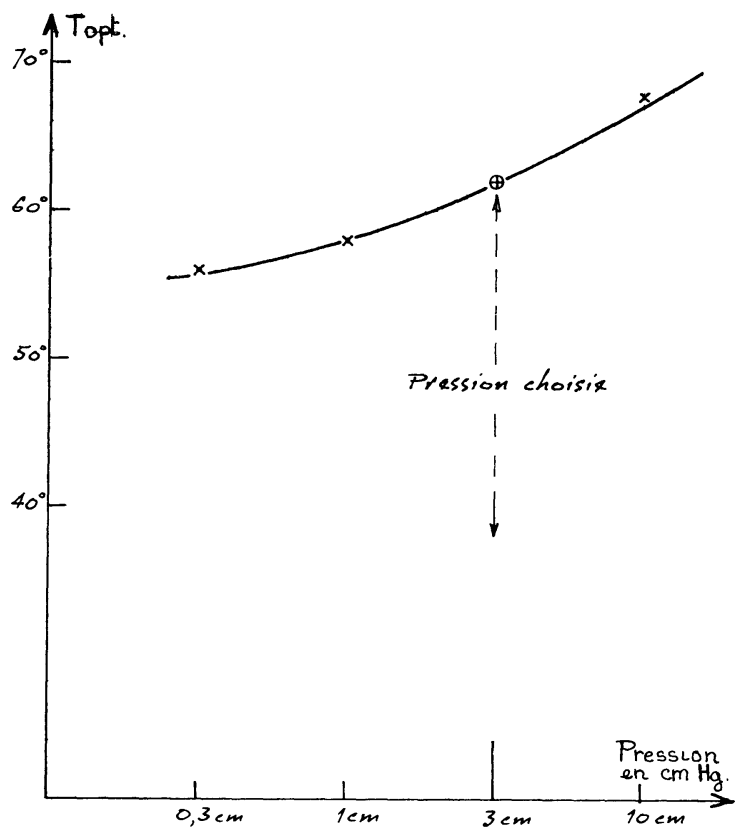

Fig. 16. - Température optimale

fonction de la pression d'azote. Ballons de $20 \mathrm{~mm}$.

tension de vapeur de rubidium que le rubidium seul à $45^{\circ} \mathrm{C}(2 \mathrm{mg} \mathrm{Rb}, 16 \mathrm{mg} \mathrm{K})$.

De telles cellules contenant rubidium et potassium ou césium et potassium ont été effectivement expérimentées : les signaux de résonance sont très légèrement inférieurs aux signaux obtenus avec l'alcalin seul, la constante de temps de relaxation étant légèrement diminuée par suite des collisions d'échange entre les deux alcalins.

Cependant, les résultats sont tels qu'il est, à l'heure actuelle, tout à fait envisageable de faire fonctionner un magnétomètre à vapeur de césium à $80^{\circ} \mathrm{C}$ sans perte de performances.

IV. Source de lumière. - La source de lumière est un des éléments essentiels du magnétomètre à vapeur de césium. Sa stabilité et sa fiabilité conditionnent le bon fonctionnement de l'appareil.

IV.1. Strugture de la source. - La source se compose de :

- Une ampoule de verre cylindrique terminée par deux queusots sphériques contenant le césium. Cette ampoule est remplie également sous faible pression de xénon dont le rôle est de stabiliser le plasma.

- Une enveloppe cylindrique comportant l'enroulement d'excitation, un condensateur d'accord et une thermistance de contrôle.

- Une enveloppe extérieure concentrique isolante thermiquement.

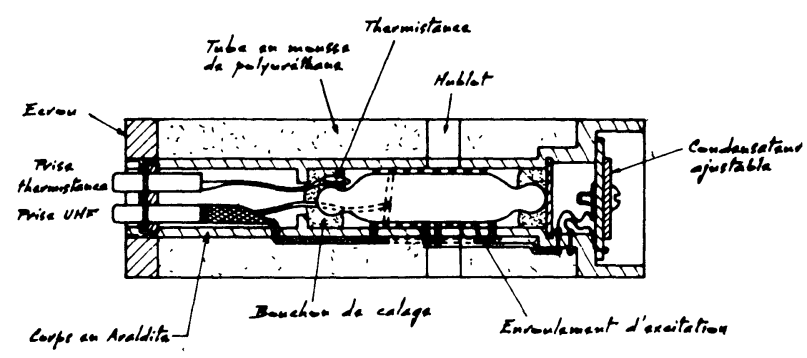

FIG. 17. - Source de lumière.

Quatre ou six ouvertures circulaires (suivant le type de sonde) permettent d'obtenir les faisceaux lumineux de pompage.

La photo 3 montre une source à 4 faisceaux : deux connecteurs coaxiaux solidaires de la source en font un élément enfichable, donc facilement interchangeable.

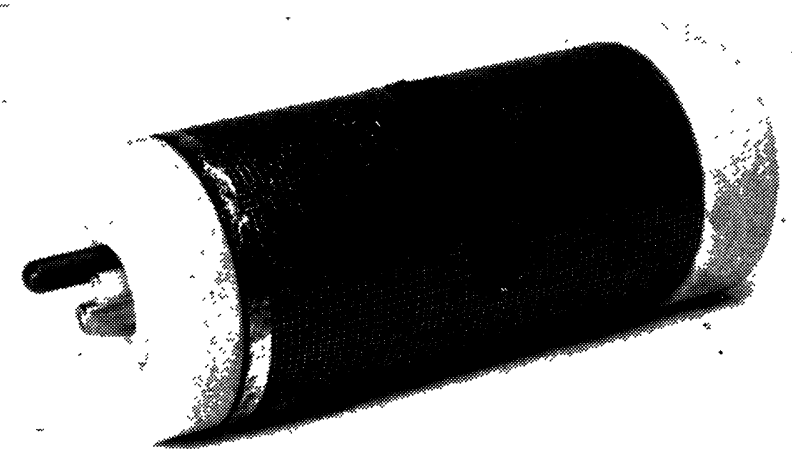

Photo 3.

IV.2. Oscillateur D'excitation de sourge. Ce circuit est un émetteur piloté par quartz dont l'étage final peut délivrer $15 \mathrm{~W}$ à $60 \mathrm{MHz}$.

La puissance de sortie est contrôlée par la thermistance de source commandant un régulateur de tension non représenté sur le schéma. Ce régulateur délivre la tension $V$ variable.

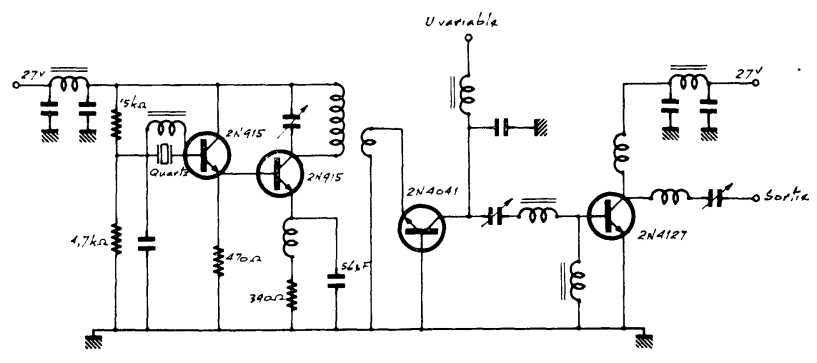

FIG. 18. - Émetteur de source.

La principale particularité de cet émetteur est son excellente stabilité à court terme qui conditionne la stabilité de la lumière.

IV.3. Fonctionnement de L'Ensemble SOURGeÉMETTEUR DE SOURGE. - L'enroulement d'excitation de la source de lumière est accordé au moyen d'un condensateur ajustable, le tout constituant un résonateur série à $60 \mathrm{MHz}$.

Le couplage se fait par prise sur l'enroulement de façon à adapter l'impédance de ce circuit à l'impédance de sortie de l'émetteur (50 ohms).

Au démarrage, la puissance incidente est de $15 \mathrm{~W}$, 


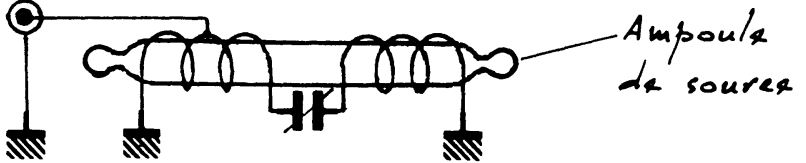

FIG. 19.

ce qui assure l'ionisation du plasma ainsi que le chauffage de l'ampoule.

La thermistance de contrôle placée sur le queusot commande ensuite cette puissance ( $§$ IV.2) de façon à maintenir la température du césium à $120^{\circ} \mathrm{C}$, ce qui correspond au maximum de rayonnement des raies $D_{1}$ et $D_{2}$ émises par le plasma.

Le temps nécessaire à la stabilisation est de l'ordre de quelques minutes. La puissance d'entretien est de $6 \mathrm{~W}$ environ.

Il faut préciser que l'ampoule de source bénéficie en fait d'une double régulation thermique.

La source se trouve à l'intérieur de la sonde qui est régulée aux environs de $60^{\circ} \mathrm{C}$ (cf. § III).

La régulation due à la thermistance de source assure donc une stabilisation fine de la température.

Cette disposition permet d'obtenir une excellente stabilité de lumière. La reproductibilité de cet ensemble a été particulièrement étudiée : il est possible, sans reprise de réglage, d'interchanger en quelques instants source ou émetteur de source sans aucune modification de performances.

La durée de vie est supérieure à 10000 heures.

V. Circuits électroniques et présentation d'ensemble. - V.1. Fonctionnement. - Le schéma de principe du magnétomètre est représenté sur la figure 20 .

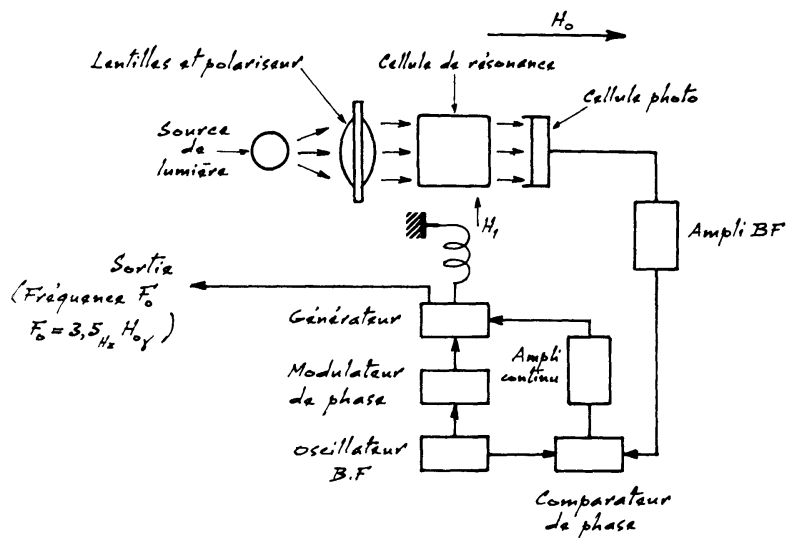

FIG. 20.

Pour faciliter la compréhension du diagramme, ce schéma ne comporte qu'une seule voie de pompage optique avec détection sur la lumière absorbée (cf. § II).

La cellule de résonance est soumise à un champ électronique $h_{1}$ de fréquence $F$ modulé en phase à la pulsation $\Omega$ :

$$
h_{1}=H_{1} \cos \left(2 \pi F t+\frac{2 \pi \Delta F}{\Omega} \cos \Omega t\right)
$$

avec $\frac{2 \pi \Delta F}{\Omega}$ excursion de phase.

Lorsque $F$ est suffisamment proche de la fréquence de résonance $F_{0}$, l'intensité de lumière $I$ détectée par la cellule photo est modulée à la pulsation $\Omega$ (fig. 21).

La tension $v=k I_{0} \cos \Omega t$ issue de la cellule est

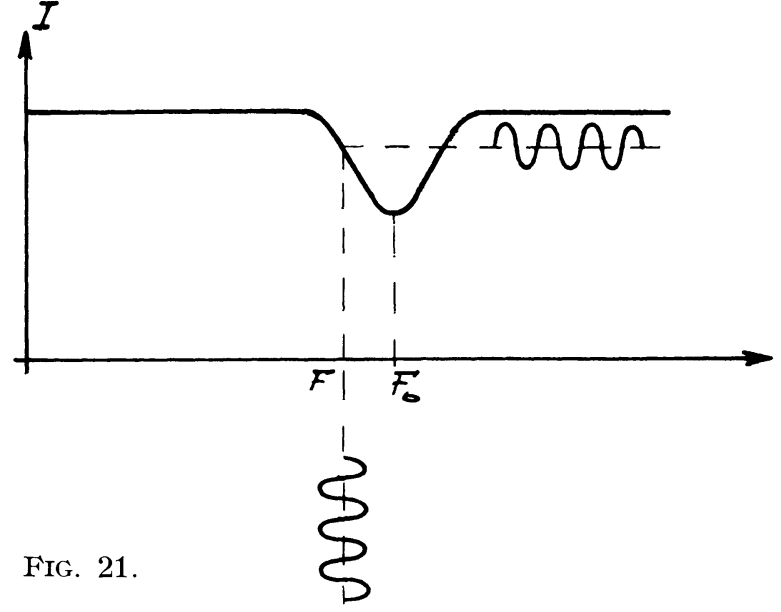

alors amplifiée puis comparée en phase avec la tension de modulation. La différence de phase varie de $180^{\circ}$ suivant que $F$ est inférieur ou supérieur à $F_{0}$. Le comparateur de phase délivre alors une tension positive ou négative qui s'annule lorsque $F=F_{0}$.

C'est ce signal d'erreur qui, amplifié, vient piloter le générateur de façon à le maintenir en permanence au centre de la raie de résonance.

La sortie du magnétomètre est donc une tension $e$ telle que :

$$
e=E \cos 2 \pi F_{0} t \quad \text { avec } \quad F_{0}=\gamma H_{0} .
$$

V.2. Description et PRÉSENTAtion DE L'APPAREIL. - L'appareil comprend une partie « détecteur » et une partie coffret électronique :

A. Détecteur. - G'est un ensemble constitué de la sonde ( 4 ou 6 faisceaux) et de son électronique associée fixées sur une « poutre » en stratifié de verre ( fig. 22 et photo 4). Cet ensemble détecteur est destiné à être monté dans un « oiseau » tracté par un avion ou un hélicoptère ou dans un « poisson » tracté par un bateau. On peut également installer l'ensemble dans la queue d'un avion conçu pour les mesures magnétiques (Bréguet 1150 par exemple).

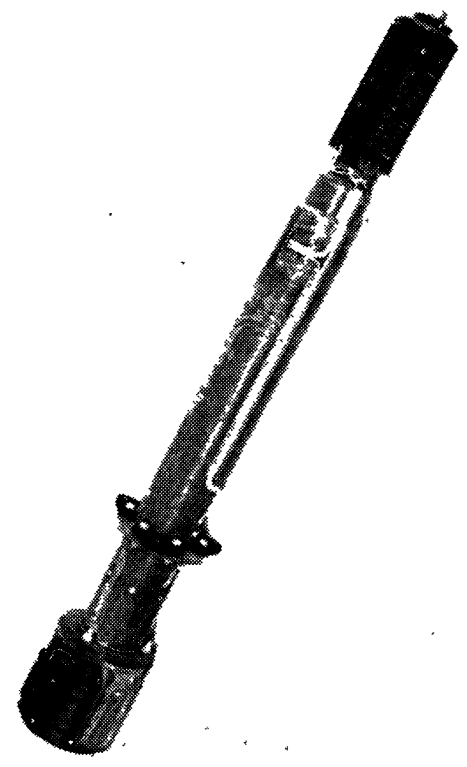

Рното 4. 


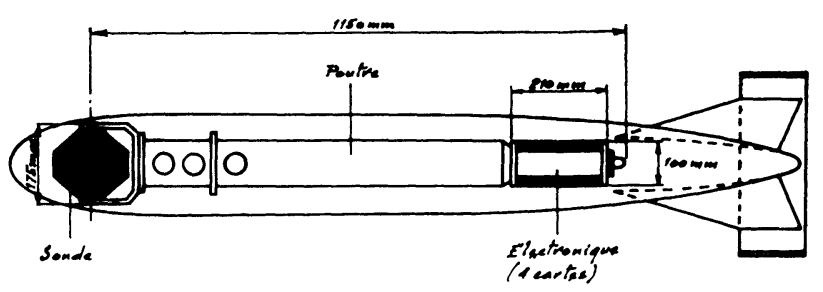

FIG. 22. - Le poids est de 6,5 kg.

L'électronique du détecteur est constituée de 3 cartes en circuit imprimé :

- émetteur de source,

- préamplificateur basse fréquence,

- régulateur de tension.

Tous ces éléments fonctionnent entre $-40{ }^{\circ} \mathrm{C}$ et $+70^{\circ} \mathrm{C}$.

B. Coffret électronique. - Le coffret électronique installé à bord de l'avion se présente sous la forme d'un rack standard 3 unités ( fig. 23).

Il comprend :

- les organes de commande : mise en route, contrôle, etc.,

- 12 cartes constituant les circuits d'asservissement,

- le thermostat de sonde,

- l'alimentation.

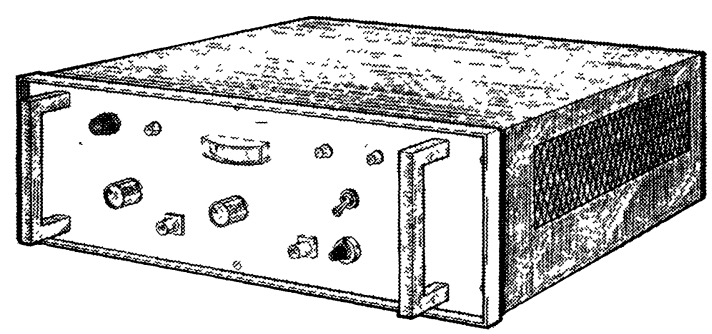

FIG. 23. - Coffret électronique.

Les circuits d'asservissement sont réalisés en grande partie en circuits intégrés série militaire. La gamme de fonctionnement en température est également comprise entre $-40^{\circ} \mathrm{C}$ et $+70^{\circ} \mathrm{C}$.

Toutes les cartes ont été conçues pour être interchangeables sans reprise de réglage.

Un balayage automatique de toute la dynamique de l'appareil (20000 à $70000 \gamma)$ s'effectue avec une récurrence de $10 \mathrm{~s}$ pendant la phase de mise en route. Dès que les températures convenables de sonde et source sont atteintes, le magnétomètre s'asservit automatiquement et le balayage est déconnecté.

L'appareil donne ses performances normales au bout de 6 à $7 \mathrm{mn}$ pour une température ambiante de $20^{\circ} \mathrm{C}$. Une position préchauffage sonde permet de réduire ce temps à $2 \mathrm{mn}$ (cette position ne met sous tension que le thermostat sonde : la source de lumière n'est pas excitée).

La fréquence $F_{0}=\gamma H_{0}(\gamma=3,5 \mathrm{~Hz}$ par gamma $)$ est multipliée par 28 dans toute la dynamique, ce qui donne une résolution de $1 \mathrm{~Hz}$ pour $0,01 \gamma$.

C. Câble de traction. - G'est un câble avec âme tractrice en acier amagnétique. Ce câble comporte 7 coaxiaux. Sa charge à la rupture est de $800 \mathrm{~kg}$. Son diamètre extérieur est de $11,5 \pm 0,25 \mathrm{~mm}$. Ce câble a une longueur de $100 \mathrm{~m}$ et il est prévu pour la traction des oiseaux derrière avion ou hélicoptère. Dans le cas de la version " poisson », le câble est à déterminer en fonction des caractéristiques du poisson. Sa longueur dépendra du magnétisme du bateau tracteur.

V.3. Organes D'explottation. - La sortie du magnétomètre peut être traitée au moyen d'un fréquencemètre classique $10 \mathrm{MHz}$ suivi soit d'un ensemble convertisseur numérique analogique avec enregistreur, soit d'une imprimante.

La résolution alors obtenue est :

$-1 \gamma$ pour toute l'échelle de l'enregistreur connecté au convertisseur numérique analogique,

$-0,01 \gamma$ par unité sur le dernier chiffre imprimé.

De nombreux constructeurs d'appareils de mesure peuvent fournir cet équipement qui est standard : Rochar, Hewlett-Packard, Monsanto, etc.

A titre d'exemple, on peut monter dans un même rack $3 \mathrm{U}$ :

- 1 compteur Rochar type A1439 et 1 convertisseur numérique analogique type A1446,

ou bien :

- 1 compteur Monsanto type 101 A et 1 convertisseur numérique analogique type $503 \mathrm{~A}$.

VI. Conclusion. - Le magnétomètre actuel à vapeur de césium type asservi est un appareil évolué bénéficiant de l'expérience acquise au cours de son industrialisation (plus de 100 équipements déjà construits).

Ses caractéristiques et performances :

- sensibilité $0,01 \gamma$,

- dynamique 20000 à $70000 \gamma$ en une seule gamme,

- effet d'orientation $<0,004 \gamma$ par degré,

- gamme de température $-40^{\circ} \mathrm{C}$ à $+50^{\circ} \mathrm{C}$,

en font un appareil adapté aussi bien aux mesures en valeur absolue (relevé des cartes magnétiques, prospection pétrolière ou minière) qu'aux mesures relatives (détection de submersible).

Les différentes études qui ont permis d'aboutir à cette réalisation ont été menées avec le concours et le soutien de la Marine Nationale Française, de la D.R.M.E. et de la Compagnie Générale de Géophysique.

\section{BIBLIOGRAPHIE}

[1] Kasther (A.), J. Physique, 1950, II, 255.

[2] MaLnar (L.) et Griveit (P.), Measurement of Weak Magnetic Fields by Magnetic Resonance, $A d v$. in Electronics and Electron Physics, 23.

[3] MeiLiferoux (J. L.), Mesucora 67, Comptes Rendus, 18 -B $3 / 1$ à 9 .
[4] Mainar (L.) et Meillekoux (J. L.), Brevet C.S.F., no 1500385 .

[5] MaLnaR (L.), Optimisation de la sensibilité d'un magnétomètre à pompage. optique (Compte Rendu de la 3 e Conférence Internationale d'Électronique Quantique, p. 306 à 315 incluse).

[6] Brun (H.), Brevet C.S.F., no 1567163. 